\title{
AVALIAÇÃo DE MÉTODOS PARA ESTIMATIVA DA DISPONIBILIDADE DE FÓSFORO PARA ARROZ EM SOLOS DE VÁRZEA DO RIO GRANDE DO SUL ${ }^{(1)}$
}

\author{
Leandro Souza da Silva ${ }^{(2)}$, Sidnei Kuster Ranno ${ }^{(3)}$, Anderson \\ Clayton Rhoden ${ }^{(4)}$, Danilo Rheinheimer dos Santos $^{(5)}$ \& Fábio \\ Adriano Graupe ${ }^{(6)}$
}

\begin{abstract}
RESUMO
A eficácia da análise de solo depende de quanto o valor obtido pelo método está correlacionado com a quantidade do nutriente absorvida pela planta, em solos de uma determinada região de utilização do sistema de recomendação de adubação. O objetivo deste trabalho foi avaliar a correlação entre o teor de $\mathbf{P}$ no solo avaliado por diferentes métodos de extração e a quantidade de $P$ absorvida por plantas de arroz irrigado por inundação. Foram coletadas amostras na camada superficial $(0-20 \mathrm{~cm})$ de solos de várzea em 16 locais do Rio Grande do Sul (RS), com ampla faixa de variação de características químicas e físicas. Os solos receberam três níveis de $P$ : $P_{0}$ (sem $P$ ), $P_{1}\left(1 / 2\right.$ de $P_{2}$ ) e $P_{2}$ (quantidade para atingir $0,2 \mathrm{mg} \mathrm{L}^{-1}$ na solução do solo, com base na capacidade máxima de adsorção de $P$ de cada solo). As unidades experimentais constaram de quatro quilogramas de solo em vasos com capacidade de $8 \mathrm{~L}$, cultivados com arroz irrigado em casa de vegetação, com três repetições e dispostos inteiramente ao acaso. Cultivou-se o arroz durante 45 dias, quando foi colhida a parte aérea das plantas e quantificado o $P$ absorvido. Amostras dos solos foram submetidas a diferentes métodos de análise de P: Mehlich-1, Mehlich-2, Mehlich-3, Resina de troca de ânions (RTA) em lâminas, RTA em esferas, Bray-1, Bray-2, Olsen, Morgan, Lactato de Ca, Texas, Oxalato,
\end{abstract}

\footnotetext{
(1) Parte da Tese de Mestrado do segundo autor apresentada ao Programa de Pós-Graduação em Ciência do Solo, Universidade Federal de Santa Maria - PPGCS/UFSM. Recebido para publicação em fevereiro de 2006 e aprovado em setembro de 2007.

(2) Professor Adjunto do Departamento de Solos da Universidade Federal de Santa Maria - UFSM. Campus Universitário, CEP 97105-900 Santa Maria (RS). E-mail: leandro@smail.ufsm.br

(3) Pesquisador da Fundação MS. Caixa Postal 105, CEP 79150-000 Maracaju (MS). E-mail: sidneiranno@yahoo.com.br

(4) Engenheiro-Agrônomo, M.Sc., Projeto PRAPEM/Microbacias 2. CEP 89896-000 Itapiranga (SC). E-mail: andersonrhoden@mail.ufsm.br

(5) Professor Adjunto do Departamento de Solos, UFSM. Bolsista do CNPq. E-mail: danilor@smail.ufsm.br

(6) Engenheiro-Agrônomo, BR 20 km 70 CEP 47650-000 Correntina (Ba). Email: graupe@mail.ufsm.br
} 
EDTA, $\mathrm{H}_{2} \mathrm{SO}_{4}$ e NaOH. Realizaram-se análises de correlação entre os teores de $\mathrm{P}$ no solo e as quantidades absorvidas pelas plantas. Dentre os métodos testados, $\mathrm{H}_{2} \mathrm{SO}_{4}$, Texas, Lactato de Ca, EDTA, Olsen e RTA foram os que se destacaram, mas apresentaram eficácia semelhante à do extrator Mehlich-1.

Termos de indexação: análise do solo, métodos, correlação, calibração.

\title{
SUMMARY: ESTIMATION METHODS OF PHOSPHORUS AVAILABILITY FOR RICE IN FLOODED SOILS IN RIO GRANDE DO SUL, BRAZIL
}

\begin{abstract}
The reliability of soil analysis depends on the correlation of nutrient concentration in the soil obtained by a given method with the plant nutrient uptake. The objective of this study was to evaluate the correlations between phosphorus content estimated by different methods and phosphorus quantity absorbed by rice plants in some flooded soils from Rio Grande do Sul state (RS), Brazil. Soil samples from 16 locations in RS, largely different in soil chemical and physical properties, were collected from the top layer $(0-20 \mathrm{~cm})$. Each soil was treated with three $P$ levels: $P_{0}$ (without $\left.P\right) ; P_{1}(1 / 2 P 2)$ and $P_{2}(P$ dose sufficient to obtain $0.2 \mathrm{mg} \mathrm{L}^{-1}$ of $P$ in soil solution, based on the maximum phosphorus adsorption capacity of each soil). The pots used to cultivate rice plants in a greenhouse were arranged in a completely randomized design, with three replications. Rice shoots were cut 45 days after emergence to evaluate $P$ uptake. The soil phosphorus concentration was evaluated by the following methods: Mehlich-1, Mehlich-2, Mehlich-3, anion exchange resin in sheets and spheres (AER), Bray-1, Bray-2, Olsen, Morgan, Calcium Lactate, Texas, Oxalate, EDTA, $\mathrm{H}_{2} \mathrm{SO}_{4}$, and $\mathrm{NaOH}$. The soil $\mathrm{P}$ concentration by the distinct extractors were compared with the $P$ absorbed by plants through linear correlation analysis. Among these methods, $\mathrm{H}_{2} \mathrm{SO}_{4}$, Texas, Calcium Lactate, EDTA, Olsen and Resin were the best, but their efficacy was similar to that of Mehlich-1.
\end{abstract}

Index terms: calibration, correlation, methods, soil analysis.

\section{INTRODUÇÃO}

O fósforo $(\mathrm{P})$ é apontado como um dos nutrientes mais limitantes ao rendimento das plantas, apesar de sua relativa abundância na crosta terrestre. De modo geral, mesmo que o teor total de P no solo seja alto em relação ao necessário para as plantas, apenas uma pequena fração deste tem baixa energia de ligação que possibilite sua dessorção dos colóides e disponibilidade às plantas. Quando o solo não tem capacidade de suprir as quantidades de Pexigidas pelas plantas e houver retorno econômico, a prática da adubação deve ser empregada. A forma mais rápida e econômica para avaliar a disponibilidade de nutrientes no solo e determinar a quantidade de fertilizantes a ser empregada se dá por meio da análise química do solo e da consulta de tabelas de recomendação de adubação. Entretanto, a eficácia do uso da análise de solo depende de quanto o valor obtido pelo método está correlacionado com a absorção do nutriente pela planta, em solos que representam uma determinada região de utilização do sistema de recomendação de adubação (Mielniczuk, 1995).

$\mathrm{A}$ avaliação do $\mathrm{P}$ disponível às plantas é feita mediante o uso de soluções extratoras com características e princípios de extração diversos (Novais \& Smith, 1999). Os métodos procuram estimar a porção de $\mathrm{P}$ na fase sólida (fator $\mathrm{Q}$ ) passível de repor o P na solução (fator I), de modo a estimar a necessidade de suplementação do nutriente. A eficiência dos métodos depende da forma em que o nutriente está inserido em Q (formas de $\mathrm{P}$ tipo Al-P, $\mathrm{Ca}-\mathrm{P}, \mathrm{Fe}-\mathrm{P}$ ) e do acesso do extrator a essa forma (Gatiboni, 2003). Nas últimas décadas, vários métodos têm sido desenvolvidos no intuito de avaliar a disponibilidade de $P$ às plantas, sendo eles classificados em categorias, conforme Kuo (1996): água ou soluções salinas não-tamponadas; ácidos fortes diluídos com a presença ou não de agentes complexantes; ácidos fracos diluídos com a presença ou não de agentes complexantes; soluções alcalinas tamponadas com a presença ou não de agentes complexantes; resina trocadora de ânions (RTA) ou tiras de papel-filtro impregnado com óxidos de $\mathrm{Fe}$; e troca isotópica com ${ }^{32} \mathrm{P}$.

Embora pareça vantajosa a existência de grande quantidade de métodos de estimativa da disponibilidade de $\mathrm{P}$ às plantas, na verdade, isso está relacionado à complexidade do comportamento do P no solo (Larsen, 1967) e à dificuldade de se obter um método satisfatório para grande número de solos e regiões. Dessa forma, a literatura aponta controvérsias quanto ao método que apresenta maior eficácia em predizer a disponibilidade de P (Silva \& Raij, 1999). 
Os métodos para avaliação da disponibilidade de $\mathrm{P}$ não precisam, necessariamente, extrair todo o fator $\mathrm{Q}$ do solo, desde que seja mantida a proporcionalidade entre a fração extraída em diferentes tipos de solo e os estoques de P (Novais \& Smith, 1999). Isso determina a necessidade de calibração do método para correlacionar o teor no solo com a absorção de $\mathrm{P}$ e a produtividade das culturas em condições de cultivo a campo. De acordo com a Comissão de Química e Fertilidade do Solo (CQFSRS/SC, 2004), o método atualmente empregado no sistema de recomendações de calagem e de adubação para o Rio Grande do Sul (RS) e Santa Catarina (SC) é o Mehlich-1. Nesse sistema, a interpretação do teor extraído considera o teor de argila em quatro classes texturais $(>60 \%=1$; 41 a $60 \%=2 ; 21$ a $40 \%=3 ;$ e $<20 \%=4$ ), cada uma com seu respectivo nível crítico, enquanto os solos de várzea sob cultivo de arroz irrigado por inundação foram agrupados na classe 5 , sem considerar o efeito da textura na interpretação do teor extraído.

Entretanto, os vários métodos de extração química que podem ser utilizados para avaliar a disponibilidade de $\mathrm{P}$ e recomendar adubação fosfatada para culturas em condições de sequeiro, geralmente, apresentam baixa eficiência em solos de várzea, tendo pouca aplicabilidade quando este solo é utilizado para cultivo de arroz sob alagamento (Patrick Jr. \& Mahapatra, 1968; Chang, 1978). O principal motivo para esse comportamento é o fato de que a extração do $\mathrm{P}$ em amostras de solo seco não prevê as alterações na quantidade de $\mathrm{P}$ disponível com as reações de redução que ocorrem no solo, proporcionadas pelo alagamento, cuja intensidade varia de solo para solo. Este parece ser o caso do método Mehlich-1, pois, segundo Grande et al. (1986), ele realiza a extração em valores de $\mathrm{pH}$ muito baixos (aproximadamente 2,5) - ambiente desfavorável para estimar as frações de $\mathrm{P}$ disponibilizadas com o alagamento do solo. Do mesmo modo, isso estaria relacionado ao fato de que os extratores ácidos dissolvem predominantemente $\mathrm{Ca}$ $\mathrm{Pe} \mathrm{Al}-\mathrm{P}$ e de que a liberação do $\mathrm{P}$ ligado ao $\mathrm{Fe}(\mathrm{Fe}-\mathrm{P})$ é o processo que predominaria em condições de alagamento do solo (Patrick Jr. \& Mahapatra, 1968). Em razão desses e outros aspectos, nos Estados do RS e SC, é crescente o número de pesquisadores que defendem o uso de outros métodos em substituição ao Mehlich-1 para avaliação da disponibilidade de $\mathrm{P}$ às plantas, mesmo para culturas de sequeiro (Gatiboni, 2003). Entre os métodos mais cogitados pela CQFSRS/ SC (2001) como substituintes do Mehlich-1 estão a RTA e o Mehlich-3, sobre os quais há pouca informação acerca do comportamento em solos de várzea cultivados com arroz irrigado por inundação.

O objetivo deste trabalho foi avaliar diferentes métodos de extração de $\mathrm{P}$ por meio da correlação entre o teor de P no solo e a quantidade absorvida pelo arroz, comparando com o método atualmente empregado na predição da disponibilidade de $\mathrm{P}$ para a cultura do arroz irrigado por alagamento nos solos do RS.

\section{MATERIAL E MÉTODOS}

Foram coletadas amostras de 16 solos de várzea representativos da lavoura orizícola do RS, significativas para produção de arroz, com ampla faixa de variação de características químicas e físicas, as quais incluem 14 municípios: Camaquã, Torres, Dom Pedrito, Uruguaiana (duas amostras), Caçapava do Sul, Santo Antônio da Patrulha, Santa Maria, São Gabriel, Cachoeirinha, Restinga Seca, Cachoeira do Sul (duas amostras), Santa Vitória do Palmar, Rosário do Sul e Paraíso do Sul. A associação entre os locais de coleta dos solos e a classificação brasileira foi efetuada de acordo com Streck et al. (2002); tendo em vista a semelhança de alguns tipos de solos, foi utilizada na discussão a denominação dos solos conforme o município de onde foram coletados (Quadro 1).

As amostras foram coletadas na camada superficial (0-20 cm). Logo em seguida, o solo foi seco ao ar, moído e peneirado a $2 \mathrm{~mm}$. Separou-se uma subamostra de cada solo para as análises de argila, areia e silte, segundo Embrapa (1997), e químicas teor de carbono orgânico e frações de Fe ditionito e oxalato, conforme Tedesco et al. (1995), Mehra \& Jackson (1960) e Schwertmann (1964), respectivamente - e para determinação da capacidade máxima de adsorção de $\mathrm{P}$ (CMAP), com base na equação de Langmuir, proposta em 1918 (Sparks, 1995), cujos detalhes operacionais estão descritos em Ranno et al. (2007) (Quadro 1). A partir da CMAP de cada solo, foram criados três níveis de $\mathrm{P}: \mathrm{P}_{0}$ (sem aplicação de $\mathrm{P}) ; \mathrm{P}_{1}$ (1/2 da quantidade de $\mathrm{P}$ aplicada no $\mathrm{P}_{2}$ ); e $\mathrm{P}_{2}$ (quantidade de $\mathrm{P}$ para atingir $0,2 \mathrm{mg} \mathrm{L}^{-1}$ de $\mathrm{P}$ na solução do solo) (Quadro 1), através da adição de fosfato de potássio monobásico $\left(\mathrm{KH}_{2} \mathrm{PO}_{4}\right)$. $\mathrm{O}$ teor de potássio nas amostras foi uniformizado com a aplicação de cloreto de potássio (KCl), de modo a complementar a diferença em relação à quantidade de $\mathrm{K}$ adicionada com o $\mathrm{KH}_{2} \mathrm{PO}_{4}$ no maior nível de $\mathrm{P}$.

Alíquotas de $4 \mathrm{~kg}$ das amostras dos solos, em seus respectivos níveis de $\mathrm{P}$, foram transferidas para vasos plásticos de $8 \mathrm{~L}$, onde se iniciou o cultivo do arroz irrigado em casa de vegetação. Utilizaram-se sementes da variedade IRGA 417, as quais foram prégerminadas durante um período de cinco dias em bandejas com papel-toalha sob alta umidade e temperatura na faixa de $20-25{ }^{\circ} \mathrm{C}$. Foram transplantadas oito plântulas para cada vaso e, quatro dias após o transplante, realizou-se a aplicação de uma lâmina de água de aproximadamente $5 \mathrm{~cm}$, a qual foi mantida constante durante o período de cultivo por meio de irrigações diárias, utilizando-se água destilada. Nove dias após o transplante, realizou-se um desbaste para manutenção de seis plantas por vaso. Neste dia, aplicou-se $\mathrm{N}$ em cobertura em dose equivalente a $17 \mathrm{mg} \mathrm{kg}^{-1}$ de $\mathrm{N}$, na forma de uréia. Realizaram-se mais três aplicações de $\mathrm{N}$ em cobertura, com a mesma dose, totalizando $68 \mathrm{mg} \mathrm{kg}^{-1} \mathrm{de} \mathrm{N}$. No 
Quadro 1. Associação entre os locais de coleta dos solos objetos de estudo e as unidades de mapeamento e classificação brasileira e atributos químicos e físicos dos solos coletados nos 16 municípios do RS

\begin{tabular}{|c|c|c|c|c|c|c|c|c|c|}
\hline \multirow{2}{*}{ Município } & \multirow{2}{*}{$\begin{array}{l}\text { Unidade de } \\
\text { mapeamento }\end{array}$} & \multirow{2}{*}{$\begin{array}{c}\text { Classificação } \\
\text { brasileira }\end{array}$} & \multirow{2}{*}{ Argila } & \multirow{2}{*}{ MO } & \multicolumn{2}{|c|}{ Fe Extraível } & \multirow{2}{*}{$\mathrm{Fe}_{\mathrm{o}} / \mathrm{Fe}_{\mathrm{d}}$} & \multirow{2}{*}{ CMAP $^{(1)}$} & \multirow{2}{*}{$\begin{array}{c}\text { Quantidade } \\
\text { de } \mathbf{P}^{(2)}\end{array}$} \\
\hline & & & & & $\mathrm{Fe}_{\mathrm{d}}$ & $\mathbf{F e}_{\mathrm{o}}$ & & & \\
\hline & & & & & $-\mathrm{g} \mathrm{kg}$ & & {[} & . $\mathrm{mg}$ & $\mathrm{kg}^{-1}$ \\
\hline Camaquã & Itapeva & Gleissolo Melânico & 804 & 70 & 10,4 & 8,5 & 0,82 & 933 & 70,8 \\
\hline Torres & Colégio & Gleissolo Melânico & 680 & 80 & 13,2 & 9,7 & 0,74 & 696 & 149,9 \\
\hline Dom Pedrito & Banhado & Gleissolo Háplico & 439 & 19 & 12,6 & 8,2 & 0,65 & 351 & 32,2 \\
\hline Uruguaiana 1 & Escobar & Vertissolo Ebânico & 370 & 55 & 34,2 & 11,8 & 0,35 & 630 & 33,6 \\
\hline Uruguaiana 2 & Uruguaiana & Chernossolo Ebânico & 330 & 36 & 13,5 & 8,7 & 0,64 & 396 & 25,6 \\
\hline Caçapava do Sul & São Gabriel & Planossolo Háplico & 320 & 29 & 6,1 & 5,1 & 0,83 & 117 & 27,8 \\
\hline $\begin{array}{l}\text { Santo Antônio } \\
\text { da Patrulha }\end{array}$ & Vacacaí & Planossolo Hidromórfico & 225 & 23 & 4,6 & 4,0 & 0,88 & 231 & 36,4 \\
\hline Santa Maria & Vacacaí & Planossolo Hidromórfico & 240 & 19 & 7,6 & 6,1 & 0,80 & 347 & 37,4 \\
\hline São Gabriel & São Gabriel & Planossolo Háplico & 245 & 26 & 18,1 & 9,3 & 0,52 & 593 & 22,3 \\
\hline Cachoeirinha & Banhado & Gleissolo Háplico & 200 & 22 & 3,9 & 3,4 & 0,86 & 252 & 15,5 \\
\hline Restinga Seca & Vacacaí & Planossolo Hidromórfico & 187 & 16 & 10,2 & 3,8 & 0,37 & 494 & 63,7 \\
\hline $\begin{array}{l}\text { Cachoeira do } \\
\text { Sul } 1\end{array}$ & Vacacaí & Planossolo Hidromórfico & 175 & 14 & 8,8 & 4,9 & 0,55 & 220 & 16,2 \\
\hline $\begin{array}{l}\text { Cachoeira do } \\
\text { Sul } 2\end{array}$ & Vacacaí & Planossolo Hidromórfico & 129 & 10 & 2,9 & 2,6 & 0,89 & 140 & 9,8 \\
\hline $\begin{array}{l}\text { Santa Vitória do } \\
\text { Palmar }\end{array}$ & Pelotas & Planossolo Hidromórfico & 123 & 19 & 1,4 & 1,1 & 0,78 & 90 & 5,9 \\
\hline Rosário do Sul & São Gabriel & Planossolo Háplico & 83 & 12 & 1,1 & 0,6 & 0,60 & 71 & 7,2 \\
\hline Paraíso do Sul & Vacacaí & Planossolo Hidromórfico & 88 & 14 & 3,1 & 1,8 & 0,58 & 236 & 11,9 \\
\hline
\end{tabular}

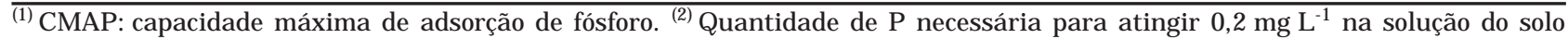
estimada a partir da CMAP de cada solo.

início do cultivo foi feita, também, aplicação de micronutrientes, cujas fontes e doses de sal aplicadas foram: ácido bórico $\left(4 \mathrm{mg} \mathrm{kg}^{-1}\right)$, sulfato de cobre (3 $\left.\mathrm{mg} \mathrm{kg}^{-1}\right)$, cloreto de zinco $\left(1 \mathrm{mg} \mathrm{kg}^{-1}\right)$ e molibdato de amônio $\left(0,15 \mathrm{mg} \mathrm{kg}^{-1}\right)$.

Cultivou-se o arroz durante 45 dias após o transplante, quando se colheu a parte aérea das plantas com o corte rente ao solo. Imediatamente após o corte, a matéria verde da parte aérea foi pesada e o material vegetal seco em estufa a $60^{\circ} \mathrm{C}$ com ventilação forçada, onde permaneceu até peso constante. Determinou-se a matéria seca e, posteriormente, realizou-se a moagem do tecido em moinho tipo Willey, no qual foi determinado o teor de $\mathrm{P}$ no tecido após digestão ácida, seguindo o método descrito por Tedesco et al. (1995). As quantidades de $\mathrm{P}$ acumuladas na parte aérea das plantas foram calculadas a partir das quantidades de matéria seca produzidas por vaso e respectivo teor de $\mathrm{P}$ na matéria seca e convertidas em quantidade de $\mathrm{P}$ absorvido pelas plantas por $\mathrm{kg}$ de solo, em função da massa de solo usada nos vasos.
Antes do cultivo do arroz irrigado, extraiu-se o $\mathrm{P}$ nas amostras de solo por diversos métodos: Mehlich-1 (Mehlich, 1953), Mehlich-2 (Mehlich, 1978), Mehlich-3 (Mehlich, 1984), Resina em lâminas (Gatiboni, 2003), Resina em esferas (Raij et al., 1987), Bray-1 (Bray \& Kurtz, 1945), Bray-2 (Bray \& Kurtz, 1945), Olsen (Tran et al., 1990), Morgan (Baker \& Hall, 1967), Lactato de Ca (Steffens, 1994), Texas (Sharpley et al., 1987), Oxalato (Shahandeh et al., 1994), EDTA (Labhsetwar \& Soltanpour, 1985), $\mathrm{H}_{2} \mathrm{SO}_{4}$ (Catani \& Gargantini, 1954) e $\mathrm{NaOH}$ (Chang \& Jackson, 1957), cujos extratores e procedimentos utilizados na extração do $P$ disponível estão resumidos no quadro 2. Outros detalhes operacionais encontramse em Ranno (2004). O P inorgânico dos extratos alcalinos, como aqueles obtidos por Olsen e $\mathrm{NaOH}$, foi determinado pelo método de Dick \& Tabatabai (1977), enquanto o $\mathrm{P}$ dos demais extratos foi determinado segundo o método de Murphy \& Riley (1962).

A eficácia dos extratores foi avaliada por meio da análise de correlação linear simples de Pearson, 
Quadro 2. Resumo da composição química dos extratores e procedimento de extração do P disponível por diferentes métodos

\begin{tabular}{|c|c|c|c|c|}
\hline Método & Solução extratora ou processo & Relação $^{(1)}$ & Tempo $^{(2)}$ & Referência \\
\hline Mehlich-1 & $\mathrm{H}_{2} \mathrm{SO}_{4} 0,0125 \mathrm{~mol} \mathrm{~L}^{-1}+\mathrm{HCl} 0,05 \mathrm{~mol} \mathrm{~L}^{-1}$ & $1: 10$ & $5 \mathrm{~min}$ & Mehlich (1953) \\
\hline Mehlich-2 & 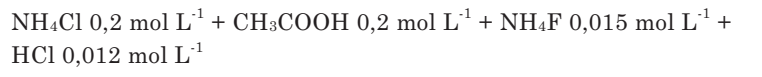 & $1: 10$ & $5 \mathrm{~min}$ & Mehlich (1978) \\
\hline Mehlich-3 & $\begin{array}{l}\mathrm{NH}_{4} \mathrm{~F} 0,015 \mathrm{~mol} \mathrm{~L}^{-1}+\mathrm{NH}_{4} \mathrm{NO}_{3} 0,25 \mathrm{~mol} \mathrm{~L}^{-1}+ \\
\mathrm{CH}_{3} \mathrm{COOH} 0,2 \mathrm{~mol} \mathrm{~L}^{-1}+\mathrm{HNO}_{3} 0,013 \mathrm{~mol} \mathrm{~L}^{-1}+\text { EDTA } 0,001 \mathrm{~mol} \mathrm{~L}^{-1}\end{array}$ & $1: 10$ & $5 \mathrm{~min}$ & Mehlich (1984) \\
\hline Resina em lâminas & Adsorção de P pela RTA saturada com bicarbonato & $1: 20$ & $16 \mathrm{~h}$ & Gatiboni (2003) \\
\hline Resina em esferas & Adsorção de P pela RTA saturada com bicarbonato & $1: 10$ & $16 \mathrm{~h}$ & Raij et al. (1987) \\
\hline Bray-1 & $\mathrm{HCl} 0,025 \mathrm{~mol} \mathrm{~L}^{-1} \mathrm{e} \mathrm{NH}_{4} \mathrm{~F} 0,03 \mathrm{~mol} \mathrm{~L}^{-1}$ & $1: 7$ & $1 \mathrm{~min}$ & Bray \& Kurtz (1945) \\
\hline Bray-2 & $\mathrm{HCl} 0,1 \mathrm{~mol} \mathrm{~L}^{-1} \mathrm{e} \mathrm{NH}_{4} \mathrm{~F} 0,03 \mathrm{~mol} \mathrm{~L}^{-1}$ & $1: 7$ & $40 \mathrm{~s}$ & Bray \& Kurtz (1945) \\
\hline Olsen & $\mathrm{NaHCO}_{3} 0,5 \mathrm{~mol} \mathrm{~L}^{-1}$ a pH 8,5 & $1: 20$ & $30 \mathrm{~min}$ & Tran et al. (1990) \\
\hline Morgan & $\mathrm{NaC}_{2} \mathrm{H}_{2} \mathrm{O}_{2} 0,735 \mathrm{~mol} \mathrm{~L}^{-1}+\mathrm{CH}_{3} \mathrm{COOH} 0,5 \mathrm{~mol} \mathrm{~L}^{-1}$ a $\mathrm{pH} 4,8$ & $1: 5$ & $30 \mathrm{~min}$ & Baker \& Hall (1967) \\
\hline Lactato de $\mathrm{Ca}$ & Lactato de $\mathrm{Ca} 0,02 \mathrm{~mol} \mathrm{~L}^{-1}+\mathrm{HCl} 0,02 \mathrm{~mol} \mathrm{~L}^{-1}$ a pH 3,7 & $1: 50$ & $90 \mathrm{~min}$ & Steffens (1994) \\
\hline Texas & $\mathrm{NH}_{4} \mathrm{Ac} 1,43 \mathrm{~mol} \mathrm{~L}^{-1}(\mathrm{pH} 4,2)+$ EDTA $0,025 \mathrm{~mol} \mathrm{~L}^{-1}$ & $1: 10$ & $30 \mathrm{~min}$ & Sharpley et al. (1987) \\
\hline Oxalato & $\left(\mathrm{NH}_{4}\right)_{2} \mathrm{C}_{2} \mathrm{O}_{4} \cdot \mathrm{H}_{2} \mathrm{O} 0,2 \mathrm{~mol} \mathrm{~L}^{-1}+\mathrm{H}_{2} \mathrm{C}_{2} \mathrm{O}_{4} 0,2 \mathrm{~mol} \mathrm{~L}^{-1}$ & $1: 40$ & $4 \mathrm{~h}$ & Shahandeh et al. (1994) \\
\hline EDTA & $\mathrm{Na}_{2}$-EDTA $0,02 \mathrm{~mol} \mathrm{~L}^{-1} \mathrm{pH} 4,8$ & $1: 25$ & $60 \mathrm{~min}$ & Labhsetwar \& Soltanpour (1985) \\
\hline $\mathrm{H}_{2} \mathrm{SO}_{4}$ & $\mathrm{H}_{2} \mathrm{SO}_{4} 0,025 \mathrm{~mol} \mathrm{~L}^{-1}$ & $1: 25$ & $15 \mathrm{~min}$ & Catani \& Gargantini (1954) \\
\hline $\mathrm{NaOH}$ & $\mathrm{NaOH} 0,1 \mathrm{~mol} \mathrm{~L}^{-1}$ & $1: 50$ & $17 \mathrm{~h}$ & Chang \& Jackson (1957) \\
\hline
\end{tabular}

${ }^{(1)}$ Massa de solo:volume de solução. ${ }^{(2)}$ Corresponde ao tempo de agitação, o qual foi seguido de período de decantação e, ou, centrifugação, em função do método, para obtenção de extrato limpo.

empregando-se a quantidade de $\mathrm{P}$ absorvida pelo arroz por $\mathrm{kg}$ de solo e o teor de $\mathrm{P}$ no solo avaliado pelos diferentes métodos. Foram consideradas as correlações com níveis de significância maiores que $99 \%(\mathrm{p}<0,01)$.

\section{RESULTADOS E DISCUSSÃo}

As quantidades de $\mathrm{P}$ absorvidas pelo arroz foram muito variáveis entre os solos, para cada um dos níveis de $\mathrm{P}$ aplicado. Sem a aplicação de $\mathrm{P}$, os valores variaram entre 0,36 e $12,24 \mathrm{mg} \mathrm{kg}^{-1}$ de solo, correspondente a uma diferença de até 34 vezes entre os solos. Mesmo comparando o maior nível de $\mathrm{P}$ em cada solo, os valores variaram entre 1,83 e $26,00 \mathrm{mg} \mathrm{kg}^{-1}$, com diferença de até 14 vezes entre solos. Essa amplitude na quantidade de $\mathrm{P}$ absorvido está relacionada à diversidade das características químicas e físicas dos solos (Quadro 1). Essa variabilidade é interessante em estudos de calibração de métodos, em que se busca uma boa estimativa da disponibilidade de $\mathrm{P}$ para todos os solos da região de abrangência do sistema de recomendação.

Para facilitar a interpretação dos resultados dos métodos avaliados, a análise de eficácia dos métodos foi feita agrupando-se aqueles que apresentavam princípios de extração de $\mathrm{P}$ semelhantes.

\section{Fósforo extraível por ácidos fortes diluídos com a presença ou não de agentes complexantes}

$\mathrm{Na}$ figura 1 estão apresentadas as correlações estabelecidas entre o teor de $\mathrm{P}$ no solo avaliado pelos extratores Mehlich-1 (1a), Bray-1 (1b), Mehlich-2 (1c), Bray-2 (1d), Mehlich-3 (1e) e $\mathrm{H}_{2} \mathrm{SO}_{4}$ (1f) e as quantidades de $\mathrm{P}$ acumuladas pela parte aérea do arroz em cada solo estudado. Obtiveram-se valores variáveis de coeficiente de correlação para este grupo de extratores, sendo o maior observado para o extrator $\mathrm{H}_{2} \mathrm{SO}_{4}\left(\mathrm{r}=0,61^{* *}\right)$, seguido por Mehlich-3 $\left(\mathrm{r}=0,47^{* *}\right)$, Mehlich-1 $\left(\mathrm{r}=0,45^{* *}\right)$, Mehlich-2 $\left(\mathrm{r}=0,44^{* *}\right)$ e Bray-2 $\left(\mathrm{r}=0,44^{* *}\right)$, com coeficientes de correlação linear simples menores, embora significativos $(\mathrm{p}<0,01)$. O método Bray-1 $\left(\mathrm{r}=0,25^{\mathrm{ns}}\right)$ foi o único extrator ácido forte cujo teor de $\mathrm{P}$ no solo não se correlacionou significativamente com a quantidade de $\mathrm{P}$ absorvido pelas plantas de arroz.

Esses resultados concordam com os de Patrick Jr. \& Mahapatra (1968), os quais afirmam que os vários métodos de extração química de $\mathrm{P}$ utilizados para avaliar a disponibilidade do nutriente em solos e recomendar adubação fosfatada para culturas em condição de sequeiro geralmente apresentam baixa eficiência em condições de solos alagados. Esse comportamento estaria relacionado ao fato de que os extratores ácidos dissolvem predominantemente $\mathrm{P}$ 


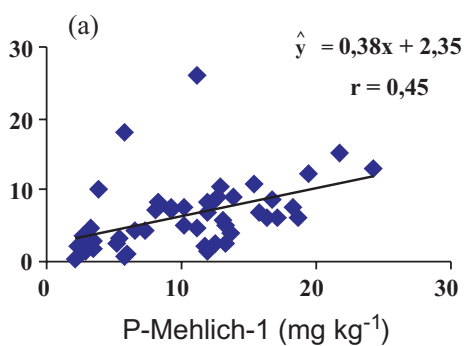

(d)
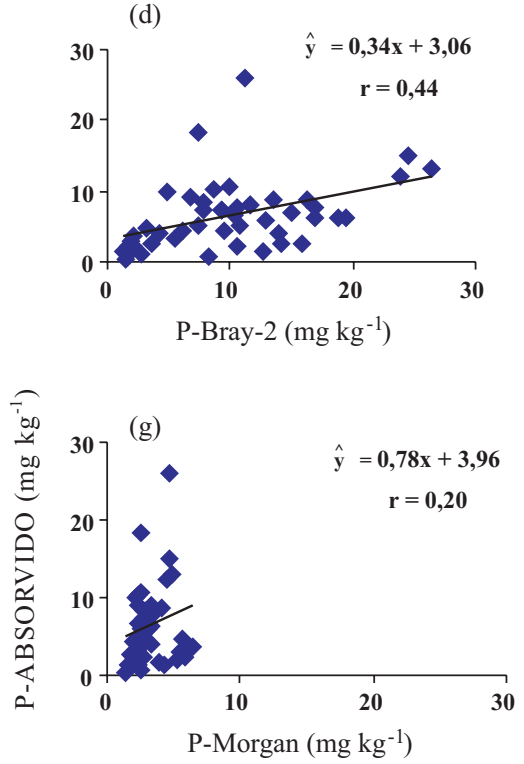

(j)

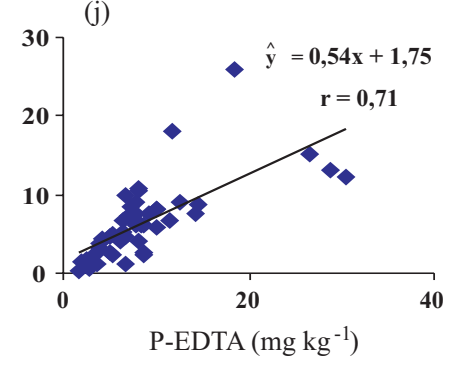

(m)

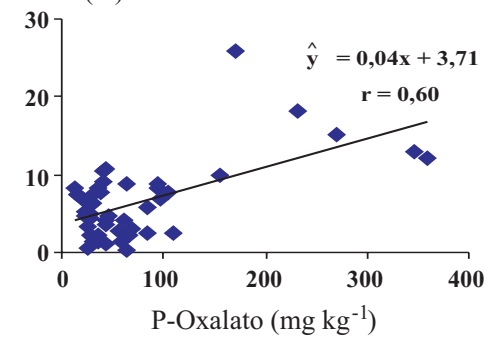

(b)

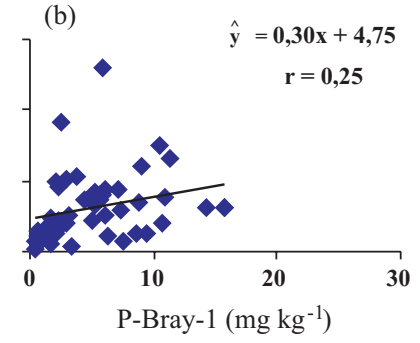

(e)
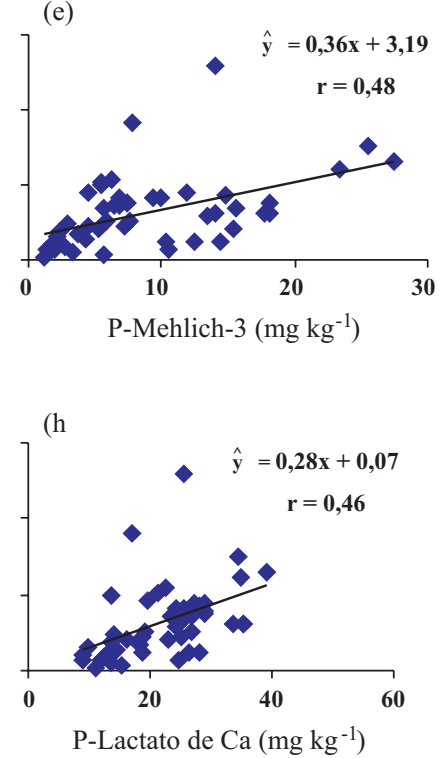

(k)

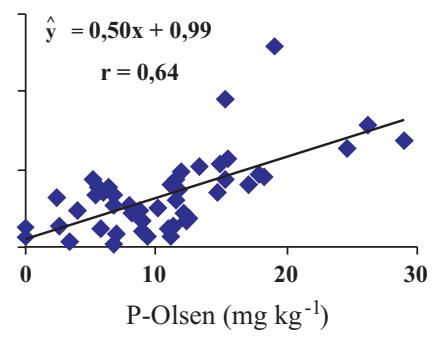

(n)

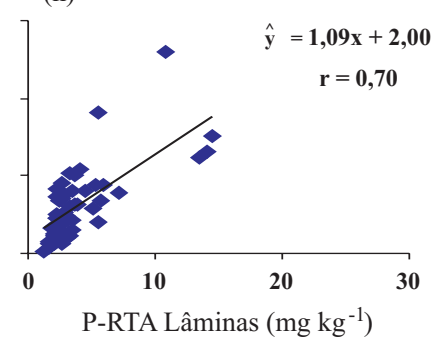

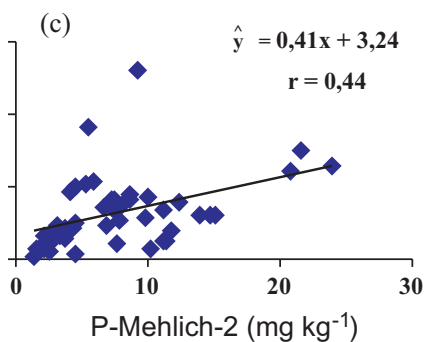
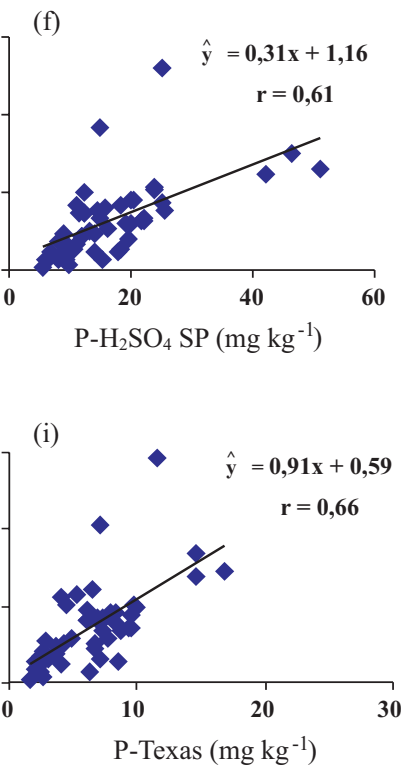

(1)

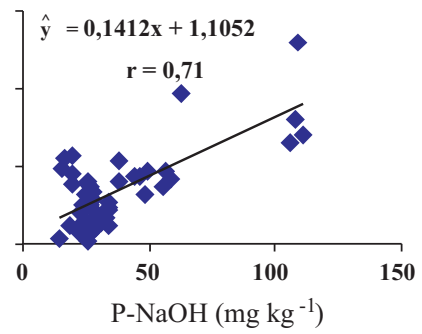

(o)

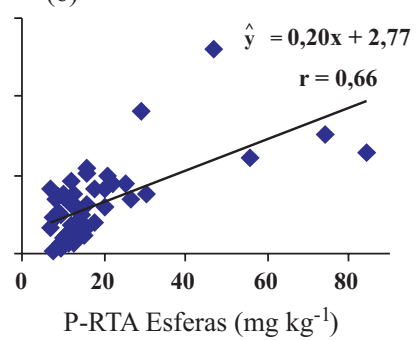

Figura 1. Correlações entre as quantidades de fósforo absorvidas pelo arroz e os teores de fósforo avaliados pelos extratores (a) Mehlich-1, (b) Bray-1, (c) Mehlich-2, (d) Bray-2, (e) Mehlich-3, (f) $\mathrm{H}_{2} \mathrm{SO}_{4}$, (g) Morgan, (h) Lactato de Ca, (i) Texas, (j) EDTA, (k) Olsen, (l) NaOH, (m) Oxalato, resina trocadora de ânions (RTA) em (n) lâminas e (o) RTA em esferas. $(n=48)$.

ligado ao Ca e ao Al, e a liberação do P ligado ao Fe é o processo que predominaria com alagamento do solo (Patrick Jr. \& Mahapatra, 1968). Neste grupo de métodos, apenas o método $\mathrm{H}_{2} \mathrm{SO}_{4}$ foi superior ao Mehlich-1 - este último é o que vem sendo atualmente empregado para avaliar a disponibilidade de $\mathrm{P}$ para 
todas as culturas nos Estados do RS e SC. Os demais métodos (Mehlich-2, Mehlich-3 e Bray-2) apresentaram coeficientes de correlação semelhantes ao obtido com Mehlich-1. A presença de íons com poder complexante, como consta em Mehlich-2, Mehlich-3, Bray-1 e Bray-2, não melhorou a correlação com o $\mathrm{P}$ absorvido, em relação à utilização simplesmente de soluções ácidas. O Mehlich-3 não apresentou maior capacidade de extração em relação ao Mehlich-1, como também foi observado por Gatiboni (2003). Isso é discordante de trabalhos cujos resultados mostram uma quantidade extraída por Mehlich-3 de 150 a $200 \%$ maior que por Mehlich-1 (Wolf \& Baker, 1985; Sims, 1989).

Analisando a distribuição dos pontos nos gráficos da figura 1, percebe-se que existem alguns deles que estão muito afastados das retas ajustadas. Esses pontos correspondem ao solo coletado no município de Torres (RS) e, conseqüentemente, contribuíram para diminuir os coeficientes de correlação obtidos para os métodos. Essa distribuição diferenciada é resultante da alta quantidade de $\mathrm{P}$ absorvido pelas plantas de arroz cultivadas neste solo, que chegou a superar em 14 vezes a quantidade absorvida pelo arroz cultivado em outros solos, mesmo quando se aplicou o maior nível de $\mathrm{P}$. Possivelmente, a quantidade de $\mathrm{P}$ que se tornou disponível com os processos de redução, devido ao alagamento, foi muito superior à dos demais solos. Todos os métodos desse grupo não foram eficazes em detectar essas alterações, pois as análises foram feitas a partir do solo seco.

Dessa forma, a exclusão do solo de Torres das análises de correlação permitiu a obtenção de melhores coeficientes de correlação linear simples entre o teor no solo e a quantidade absorvida, os quais passaram para $0,80 * *$ no $\mathrm{H}_{2} \mathrm{SO}_{4} ; 0,74 * *$ no Mehlich- 1 ; $0,67 * *$ no Bray-2; $0,65 * *$ no Mehlich-2; $0,62 * *$ no Mehlich-3; e $0,43 * *$ no Bray-1, todos significativos $(\mathrm{p}<0,01)$. Esse procedimento de exclusão é adotado em estudos de calibração de métodos quando se tem no conjunto de solos em estudo algum que apresente comportamento totalmente distinto dos demais (Menon et al., 1990).

\section{Fósforo extraível por ácidos fracos diluídos com presença ou não de agentes complexantes}

$\mathrm{Na}$ figura 1 estão apresentadas as correlações estabelecidas entre o teor de $\mathrm{P}$ no solo avaliado pelos extratores Morgan (1g), Lactato (1h), Texas (1i) e EDTA (1j) e as quantidades de $\mathrm{P}$ absorvidas pelo arroz para todos os solos. Os coeficientes de correlação também foram variados para este grupo de extratores, os quais extraem o $\mathrm{P}$ do solo em meio ácido, de modo semelhante aos anteriormente discutidos. As maiores correlações foram verificadas com os extratores EDTA $\left(\mathrm{r}=0,71^{* *}\right)$ e Texas $\left(\mathrm{r}=0,66^{* *}\right)$, seguidos do Lactato de $\mathrm{Ca}\left(0,46^{* *}\right)$, todos significativos $(\mathrm{p}<0,01)$. O teor de $\mathrm{P}$ no solo avaliado pelo extrator Morgan não se correlacionou com o $\mathrm{P}$ absorvido pelas plantas $\left(r=0,20^{\mathrm{ns}}\right)$.
Embora os extratores EDTA e Texas apresentem os maiores coeficientes de correlação entre os métodos discutidos neste trabalho, ainda se percebe o efeito atribuído ao solo de Torres sobre a reta ajustada. A exclusão do solo de Torres também permitiu a obtenção de melhores coeficientes de correlação linear simples entre o teor no solo e a quantidade absorvida neste grupo de métodos, os quais passaram a $0,80 * *$ no EDTA; 0,79** no Texas; $0,70 * *$ no Lactato de $\mathrm{Ca}$, todos eles significativos ( $p<0,01$ ); e 0,18 no Morgan, não-significativo. Os métodos EDTA e Texas, os quais já se mostravam os mais promissores considerando todos os solos, continuaram apresentando os maiores coeficientes de correlação. Esse melhor desempenho pode estar relacionado ao princípio de extração destes métodos, os quais, em função do poder de complexação de Fe do EDTA, liberam o fosfato dos grupos funcionais dos colóides inorgânicos. Esse princípio está mais bem associado às formas de $\mathrm{P}$ afetadas pela redução do solo, cuja fração Fe-P controla a maior parte do $\mathrm{P}$ disponível nos solos. Entretanto, os valores de correlação obtidos por este grupo de métodos, quando da exclusão do solo de Torres, são semelhantes aos obtidos com o método oficial adotado pela CQFSRS/SC (2004). Isso seria suficiente para justificar a manutenção do Mehlich1 , pois este é muito mais prático e barato. Para substituir um método de laboratório, também se devem considerar outros fatores, como facilidade de operação, treinamento de funcionários, custos, disponibilidade de equipamentos, etc.

\section{Fósforo extraível por soluções alcalinas tamponadas com a presença ou não de agentes complexantes}

Na figura 1 estão apresentadas as correlações estabelecidas entre o teor de $\mathrm{P}$ no solo avaliado pelos extratores Olsen (1k), $\mathrm{NaOH}$ (11) e Oxalato (1m) e as quantidades de $\mathrm{P}$ absorvidas pelo arroz para todo o conjunto de solos. A maior correlação foi verificada com o extrator $\mathrm{NaOH}\left(\mathrm{r}=0,71^{* *}\right)$, seguido por Olsen $\left(\mathrm{r}=0,64^{* *}\right)$ e Oxalato $\left(\mathrm{r}=0,60^{* *}\right)(\mathrm{p}<0,01)$. Embora o extrator $\mathrm{NaOH}$ tenha apresentado valor de r superior ao obtido por Olsen, o primeiro não mostrou adequada distribuição dos pontos ao longo do eixo x (Figura 11), o que também pode ser observado para o extrator Oxalato $(1 \mathrm{~m})$, limitando a utilização somente do coeficiente de correlação para selecionar o melhor método e a consideração de que estes seriam métodos adequados para estimativa da disponibilidade de $\mathrm{P}$ dos solos.

A exclusão dos pontos relativos ao solo de Torres permitiu a obtenção de melhores coeficientes de correlação linear simples apenas para o extrator Olsen $\left(\mathrm{r}=0,70^{* *}\right)(\mathrm{p}<0,01)$. No caso dos extratores $\mathrm{NaOH}$ e Oxalato, os coeficientes de correlação baixaram para $0,64 * *(p<0,01)$ e $0,55 * *(p<0,01)$, respectivamente, sem perder, no entanto, a significância. Esses resultados não confirmam a eficácia esperada para este grupo de extratores na predição da disponibilidade de $\mathrm{P}$ para o arroz cultivado em solos alagados. 
Teoricamente, como a extração por estes métodos ocorre em $\mathrm{pH}$ elevado, o que é favorável à extração do $\mathrm{P}$ ligado ao $\mathrm{Fe}$ (fração predominante) e com $\mathrm{pH}$ semelhante ao de equilíbrio dos solos de várzea após o alagamento (condição na qual ocorre a absorção de $\mathrm{P}$ pelo arroz) (Mahapatra \& Patrick, 1969), estes métodos deveriam apresentar maiores coeficientes de correlação entre o teor de $\mathrm{P}$ no solo e o $\mathrm{P}$ absorvido. O trabalho de Ekpete (1976) mostra resultados positivos com o extrator Olsen quanto à estimativa da disponibilidade de $\mathrm{P}$ para o arroz irrigado por alagamento, mesmo se analisando o solo seco. Shahandeh et al. (1994) obtiveram resultados favoráveis com o extrator Oxalato, preconizando a sua utilização na predição da disponibilidade de $\mathrm{P}$ em função da dissolução seletiva do $\mathrm{P}$ ligado a óxidos de $\mathrm{Fe}$ pouco cristalinos. Essa incapacidade na obtenção de estimativa adequada da disponibilidade de $\mathrm{P}$ por este grupo de métodos, a qual já foi verificada para os extratores ácidos fortes e fracos, pode estar relacionada à diversidade de solos com variadas características químicas, físicas e mineralógicas existentes no RS e, conseqüentemente, às formas e dinâmica do $\mathrm{P}$ no solo após a inundação. Provavelmente, os resultados satisfatórios obtidos pelos autores citados estejam relacionados à utilização, em seus trabalhos, de menor quantidade e diversidade de solos.

\section{Fósforo extraível por resina trocadora de ânions (RTA)}

Nas figuras $1 \mathrm{n}$ e lo estão apresentadas as correlações estabelecidas entre o teor de $\mathrm{P}$ no solo avaliado pela RTA em lâminas e esferas, respectivamente, e as quantidades de $\mathrm{P}$ absorvidas pelo arroz para todo o conjunto de solos, cujos coeficientes foram significativos $\left(\mathrm{r}=0,70^{* *} \mathrm{e} 0,66^{* *}\right.$, respectivamente) $(p<0,01)$. Esses extratores, teoricamente, extraem o P do solo de uma maneira semelhante à absorção das plantas (Silva \& Raij, 1999). A exclusão dos pontos correspondentes ao solo de Torres pouco modificou a magnitude do coeficiente de correlação linear simples para a RTA em lâminas, que aumentou para $0,73^{* *}(p<0,01)$. Cabe salientar que as quantidades de $\mathrm{P}$ extraídas pelo método das lâminas se correlacionaram com as quantidades extraídas pelo método de esferas $(r=0,96, p<0,01)$, mas extraindo aproximadamente $20 \%$ da quantidade, o que deve estar relacionado com um diferente contato resina:solo entre os métodos usados neste estudo (Quadro 2).

Considerando que nenhum dos métodos avaliados supera de modo expressivo o Mehlich-1, utilizando-se todo o conjunto de solos, a separação dos solos em grupos com características que estão relacionadas à adsorção de P (Novais \& Smith, 1999) ou com as reações de oxirredução de solos alagados (Grande et al., 1986) parece ser um procedimento inevitável para aprimorar a eficácia do Mehlich-1, ou dos outros métodos avaliados, para uso em solos de várzea do RS destinados ao cultivo de arroz irrigado por alagamento. Essa já é uma estratégia adotada para interpretação do teor extraído por Mehlich-1 de solos do RS em condições de sequeiro e permitiu a obtenção de adequada estimativa do $\mathrm{P}$-disponível às culturas cultivadas nessas condições. Assim, mesmo que o princípio de extração destes métodos não seja o mais adequado para extrair o P disponível com o alagamento do solo, sua separação em grupos de solos poderia favorecer a obtenção de adequadas correlações com a quantidade absorvida pelas plantas de arroz, produzindo-se diferentes valores de nível crítico para cada grupo de solos.

\section{CONCLUSÃo}

Os métodos $\mathrm{H}_{2} \mathrm{SO}_{4}$, Texas, Lactato de Ca, EDTA, Olsen e RTA foram os mais promissores para avaliar a disponibilidade de $\mathrm{P}$ em solos de várzea do $\mathrm{RS}$, porém se assemelharam à eficácia obtida com o extrator Mehlich-1, enquanto os demais testados apresentaram baixa eficácia. Isso significa que não há justificativa para substituição do método atualmente empregado na análise de solos destinados ao cultivo de arroz irrigado por inundação no RS, mas que outros procedimentos devem ser estudados para melhorar a predição da disponibilidade de $\mathrm{P}$ para $\mathrm{o}$ arroz cultivado em condições de alagamento do solo.

\section{AGRADECIMENTOS}

À CAPES e ao CNPq/PIBIC, pelas bolsas de estudo; aos pesquisadores Silvio A. Genro Jr., Elio Marcolin e Vera R. M. Macedo, do IRGA, pelo auxílio na coleta das amostras de solo; à pesquisadora Walkyria $\mathrm{B}$. Scivittaro, da Embrapa-CPACT, pelas análises de P por RTA em esferas; e aos acadêmicos da UFSM Fábio J. K. Mallmann e Darines Britzke, pelo auxílio na condução do experimento.

\section{LITERATURA CITADA}

BAKER, D.E. \& HALL, J.K. Measurements of phosphorus availability in acid soils of Pennsylvania. Soil Sci. Soc. Am. Proc., 31:662-667, 1967.

BRAY, R.H. \& KURTZ, L.T. Determination of total, organic and available forms of phosphorus in soils. Soil Sci., 59:39$45,1945$.

CATANI, R.A. \& GARGANTINI, H. Extração do fósforo do solo pelo método de Neubauer e por métodos químicos. Bragantia, 13:55-62, 1954.

CHANG, S.C. \& JACKSON, M.L. Fractionation of soil phosphorus. Soil Sci, 84:133-144, 1957. 
CHANG, S.C. Evaluation of the fertility of rice soils. In: INTERNATIONAL RICE RESEARCH INSTITUTE. Soil \& Rice. Los Banõs, 1978. p.521-541.

COMISSÃO DE QUÍMICA E FERTILIDADE DO SOLO CQFSRS/SC. Alterações nas recomendações de adubação e calagem para os Estados do Rio Grande do Sul e Santa Catarina. Porto Alegre, Sociedade Brasileira de Ciência do Solo/Núcleo Regional Sul, 2001. 4p.

COMISSÃO DE QUÍMICA E FERTILIDADE DO SOLO CQFSRS/SC. Manual de adubação e de calagem para os Estados do Rio Grande do Sul e Santa Catarina. Passo Fundo, Sociedade Brasileira de Ciência do Solo/Núcleo Regional Sul, 2004. 400p.

DICK, W.A. \& TABATABAI, M.A. Determination of orthophosphate in aqueous solutions containing labile organic and inorganic phosphorus compounds. J. Environ. Qual., 6:82-85, 1977.

EKPETE, D.M. Evaluation of chemical methods for the determination of available phosphorus in waterlogged soils. Soil Sci., 121:7-221, 1976.

EMPRESA BRASILEIRA DE PESQUISA AGROPECUÁRIA EMBRAPA. Centro Nacional de Pesquisa de Solos. Manual de métodos de análise de solo. Brasília, 1997. $212 \mathrm{p}$.

GATIBONI, L.C. Disponibilidade de formas de fósforo do solo às plantas. Santa Maria, Universidade Federal de Santa Maria, 2003. 231p. (Tese de Doutorado)

GRANDE, M.A.; CURI, N. \& QUAGGIO, J.A. Disponibilidade de fósforo pelos extratores de Mehlich e Resina em solos cultivados com arroz irrigado. R. Bras. Ci. Solo, 10:45-50, 1986.

KUO, S. Phosphorus. In: SPARKS, D.L.; PAGE, A.L.; HELMKE P.A.; LOEPPERT, R.H.; SOLTANPOUR, P.N.; TABATABAI, M.A.; JOHNSTON, C.T. \& SUMNER, M.E., eds. Methods of soil analysis: Chemical methods. Madison, Soil Science Society of America, 1996. p.869-920.

LABHSETWAR, V.K. \& SOLTANPOUR, P.N. A comparison of $\mathrm{NH}_{4} \mathrm{HCO}_{3}$-DTPA, $\mathrm{NaHCO}_{3}, \mathrm{CaCl}_{2}$, and $\mathrm{Na}_{2}$-EDTA soil tests for phosphorus. Soil Sci. Soc. Am. J., 49:1437-1440, 1985 .

LARSEN, S. Soil phosphorus. Adv. Agron., 19:151-210, 1967.

MAHAPATRA, I.C. \& PATRICK JR, W.H. Inorganic phosphate transformation in waterlogged soils. Soil Sci., 107:281288, 1969.

MEHLICH, A. Determination of $\mathrm{P}, \mathrm{Ca}, \mathrm{Mg}, \mathrm{K}, \mathrm{Na}$ and $\mathrm{NH}_{4}$ by North Carolina Soil Testing Laboratories. Raleigh, University of North Carolina, 1953. Não Publicado.

MEHLICH, A. Mehlich 3 soil test extractant: A modification of Mehlich 2 extractant. Commun. Soil Sci. Plant Anal., 15:1409-1416, 1984.

MEHLICH, A. New extractant for soil test evaluation of phosphorus, potassium, magnesium, calcium, sodium, manganese and zinc. Comm. Soil Sci. Plant Anal., 9:477492,1978 .
MEHRA, O.P. \& JACKSON, M.L. Iron oxide removal from soils and clays by a dithionite-citrate-bicarbonate system buffered with sodium bicarbonate. Clays Clay Miner., 7:317-327, 1960.

MENON, R.G.; CHIEN, S.H. \& HAMMOND, L.L. Development and evaluation of the $\mathrm{Pi}$ soil test for plant-available phosphorus. Comm. Soil Sci. Plant Anal., 21:1131-1150, 1990.

MIELNICZUK, J. Análises de solo e sua interpretação. In: GIANELLO, C.; BISSANI, C.A. \& TEDESCO, M.J., eds. Princípios de fertilidade do solo. Porto Alegre, Universidade Federal do Rio Grande do Sul, 1995. p.3346.

MURPHY, J. \& RILEY, J.P. A modified single solution method for the determination of phosphate in natural waters. Anal. Chim. Acta, 27:31-36, 1962.

NOVAIS, R.F. \& SMYTH, T.J. Fósforo em solo e planta em condições tropicais. Viçosa, MG, Universidade Federal de Viçosa, 1999. 399p.

PATRICK JR., W.H. \& MAHAPATRA, I.C. Transformation and availability to rice of nitrogen and phosphorus in waterlogged soils. Adv. Agron., 20:323-359, 1968.

RAIJ, B.van; QUAGGIO, J.A.; CANTARELLA, H.; FERREIRA, M.E.; LOPES, A.S. \& BATAGLIA, O.C. Análise química do solo para fins de fertilidade. Campinas, Fundação Cargill, 1987. 170p.

RANNO, S.K. Estimativa da disponibilidade de fósforo para a cultura do arroz irrigado em solos do RS. Santa Maria, Universidade Federal de Santa Maria, 2004. 139p. (Tese de Mestrado)

RANNO, S.K.; SILVA, L.S.; GATIBONI, L.C. \& RHODEN, A.C. Capacidade de adsorção de fósforo em solos de várzea do Estado do Rio Grande do Sul. R. Bras. Ci. Solo, 31:21-28, 2007.

SCHWERTMANN, U. Differenzierung der Eisenoxide des Bodens durch Extraction mit Ammonuioxalat-losung. Pflanzenerhähr Bodenk., 105:194-202, 1964.

SHAHANDEH, H.; HOSSNER, L.R. \& TURNER, F.T. Phosphorus relationships in flooded rice soils with low extractable phosphorus. Soil Sci. Soc. Am. J., 58:11841189, 1994.

SHARPLEY, A.N.; TIESSEN, H. \& COLE, C.V. Soil phosphorus forms extracted by soil tests as a function of pedogenesis. Soil Sci. Am. J., 51:362-365, 1987.

SILVA, F.C. \& RAIJ, B.van. Disponibilidade de fósforo em solos avaliada por diferentes extratores. Pesq. Agropec. Bras., 34:267-288, 1999.

SIMS, J.T. Comparison of Mehlich 1 and Mehlich 3 extractants for $\mathrm{P}, \mathrm{K}, \mathrm{Ca}, \mathrm{Mg}, \mathrm{Mn}, \mathrm{Cu}$ and $\mathrm{Zn}$ in Atlantic coastal plain soils. Comm. Soil Sci. Plant Anal., 20:1707-1726, 1989.

SPARKS, D. Environmental soil chemistry. San Diego, Academic Press, 1995. 267p.

STEFFENS, D. Phosphorus release kinetics and extractable phosphorus after long-term fertilization. Soil Sci. Soc. Am. J., 58:1702-1708, 1994. 
STRECK, E.V.; KÄMPF, N.; DALMOLIN, R.S.D.; KLAMT, E.; NASCIMENTO, P.C. \& SCHNEIDER, P. Solos do Rio Grande do Sul. Porto Alegre, Universidade Federal do Rio Grande do Sul, 2002. 107p.

TEDESCO, M.J.; GIANELLO, C.; BISSANI, C.A.; BOHNEN, H. \& VOLKWEISS, S.J. Análise de solo, plantas e outros materiais. 2.ed. Porto Alegre, Universidade Federal do Rio Grande do Sul, 1995. 174p. (Boletim Técnico, 5)
TRAN, T.S.; GIROUX, M.; GUILBEAULT, J. \& AUDESSE, P. Evaluation of Mehlich-3 extractant to estimate the available P in Quebec soils. Comm. Soil Sci. Plant Anal., $21: 1-28,1990$

WOLF, A.M. \& BAKER, D.E. Comparisons of soil test phosphorus by Olsen, Bray 1, Mehlich-1 and Mehlich-3 methods. Comm. Soil Sci. Plant Anal., 16:467-484, 1985. 